\title{
芳基桥联的二奧二酰亚胺的设计合成及场效应晶体管性能研究
}

\author{
侯斌 ${ }^{a}$ 李晶 ${ }^{a}$ 辛涵申 ${ }^{a}$ 杨笑迪*, $*^{\text {高洪䂞 }}{ }^{a}$ \\ 彭培珍 ${ }^{a}$ 高希珂 $*, a$ \\ $\left({ }^{a}\right.$ 中国科学院大学 中国科学院上海有机化学研究所 中国科学院有机功能分子合成与组装化学重点实验室 \\ 上海 200032) \\ ( ${ }^{b}$ 上海中医药大学 创新中药研究院 上海 201203)
}

\begin{abstract}
摘要 在本工作中, 发展了一种新的合成策略, 利用奧各位点的反应活性差异选择性地在真的 1-位进行官能团化, 合 成了由苯环(B)或噻吩并[3,2- $b$ 噻吩基团(TT)桥联的二奧二酰亚胺化合物 AzAzBDI-1、AzAzBDI-2 和 AzAzTTDI. AzAzBDI-2 的单晶结构显示其具有扭曲的共轭骨架, 相邻的两个分子通过分子间奧的七元环和五元环的 $\pi-\pi$ 相互作用 形成二聚体, 二聚体与二聚体之间通过强的 $\pi-\pi$ 相互作用形成滑移的一维堆积. 用紫外-可见光吸收光谱和循环伏安法 对三个化合物的光谱和电化学性质进行表征, 计算出各分子的轨道能级和带隙. 基于三个化合物的有机场效应晶体管 器件(OFET)均表现出 n-型主导的双极性有机半导体特性. 其中 AzAzTTDI 表现出最优的 OFET 性能, 其电子和空穴迁 移率分别为 $0.087 \mathrm{~cm}^{2} \cdot \mathrm{V}^{-1} \cdot \mathrm{s}^{-1}$ 和 $8.8 \times 10^{-3} \mathrm{~cm}^{2} \cdot \mathrm{V}^{-1} \cdot \mathrm{s}^{-1}$.
\end{abstract}

关键词＼cjkstart真; 非苯芳烃; 酰亚胺; 有机场效应晶体管; 有机半导体

\section{Design, Synthesis and Field Effect Characteristics of Diazulene Diimides Bridged by Aromatic Group}

\author{
Hou, Bin ${ }^{a} \quad$ Li, Jing ${ }^{a} \quad$ Xin, Hanshen $^{a} \quad$ Yang, Xiaodi* ${ }^{*} \quad$ Gao, Honglei $^{a}$ \\ Peng, Peizhen ${ }^{a} \quad$ Gao, Xike*,
}

$\left({ }^{a}\right.$ Key Laboratory of Synthetic and Self-Assembly Chemistry for Organic Functional Molecules, Center for Excellence in Molecular Synthesis, Shanghai Institute of Organic Chemistry, University of Chinese Academy of Sciences, Chinese Academy of Sciences, Shanghai 200032, China)

( ${ }^{b}$ Innovation Research Institute of Traditional Chinese Medicine, Shanghai University of Traditional Chinese Medicine, Shanghai 201203, China)

Abstract Azulene, a bicyclic nonbenzenoid aromatic hydrocarbon, shows completely different physicochemical properties compared with its isomeric naphthalene. Herein, we made use of the diverse reactivity of each position on azulene to design a new synthetic strategy for azulene-based diimides bridged by phenyl or thieno[3,2-b]thiophenyl group, 2-(azulen-2'-yl)-5-(azulen-2"-yl)benzene-1,1':4,1"-tetracarboxylic diimides (AzAzBDI-1/2) and 2-(azulen-2'-yl)-5(azulen-2"-yl)thieno[3,2-b]thiophene-3,1':6,1"-tetracarboxylic diimide (AzAzTTDI). The key step was double trifluoroacetylation at 1-position of two azulene moieties of the molecule followed by hydrolysis, anhydridization and imidization to obtain the target compounds. The single crystal structure analysis demonstrates that AzAzBDI-2 has twisted molecular backbone. The adjacent two molecules form a dimer through the intermolecular $\pi$ - $\pi$ stacking $(0.365 \mathrm{~nm})$ between the five-membered ring and the seven-membered ring of two different azulene units. Strong $\pi-\pi$ intermolecular interactions $(0.355 \mathrm{~nm})$ exist among the dimers to form a slipped one-dimensional (1D) packing motif in the crystal. For three compounds, the optoelectronic properties were investigated by UV-vis absorption spectra and cyclic voltammetry, and their energy levels of highest occupied molecular orbital (HOMO) and lowest unoccupied molecular orbital (LUMO) and the energy gaps were calculated. The HOMO/LUMO energy levels of AzAzBDI-1, AzAzBDI-2 and AzAzTTDI are $-5.56 /-3.28 \mathrm{eV},-5.56 /-3.30$ $\mathrm{eV}$ and $\quad-5.57 /-3.42 \mathrm{eV}$, respectively. The end absorptions of AzAzBDI-1, AzAzBDI-2 and AzAzTTDI in thin films show obvious red-shift $(13,13$ and $25 \mathrm{~nm})$ relative to those in $\mathrm{CHCl}_{3}$ solution, indicating strong intermolecular interactions in solid state. The charge carrier transport properties of three compounds were studied through organic field-effect transistors (OFETs). Bottom-gate and top-contact OFET devices of AzAzBDI-1, AzAzBDI-2 and AzAzTTDI were fabricated by spin-coated their respective solution on octadecyltrimethoxysilane (OTMS)-treated $\mathrm{SiO}_{2} / \mathrm{Si}$ substrates. Under nitrogen atmosphere, all of these three compounds displayed electron-dominated ambipolar organic semiconductor characteristics. The

*E-mail: yangxiaodi@shutcm.edu.cn,gaoxk@mail.sioc.ac.cn

Received May 11, 2020; published June 10, 2020.

Supporting information for this article is available free of charge via the Internet at http://sioc-journal.cn.

Project supported by the National Natural Science Foundation of China (Nos. 21522209, 21790362) and the Science and Technology Commission of Shanghai Municipality (Nos. 19XD1424700, 18JC1410600).

项目受国家自然科学基金(Nos. 21522209, 21790362)和上海市科学技术委员会项目(Nos. 19XD1424700, 18JC1410600)资助. 
electron mobilities of AzAzBDI-1 and AzAzBDI-2 were $0.068 \mathrm{~cm}^{2} \cdot \mathrm{V}^{-1} \cdot \mathrm{s}^{-1}$ and $0.086 \mathrm{~cm}^{2} \cdot \mathrm{V}^{-1} \cdot \mathrm{s}^{-1}$ and the hole mobility were $3.1 \times 10^{-4} \mathrm{~cm}^{2} \cdot \mathrm{V}^{-1} \cdot \mathrm{s}^{-1}$ and $1.5 \times 10^{-3} \mathrm{~cm}^{2} \cdot \mathrm{V}^{-1} \cdot \mathrm{s}^{-1}$, respectively. OFETs based on AzAzTTDI showed the highest electron mobility and hole mobilities of $0.087 \mathrm{~cm}^{2} \cdot \mathrm{V}^{-1} \cdot \mathrm{s}^{-1}$ and $8.8 \times 10^{-3} \mathrm{~cm}^{2} \cdot \mathrm{V}^{-1} \cdot \mathrm{s}^{-1}$, respectively. The X-ray diffraction (XRD) and atomic force microscopy (AFM) studies demonstrate thin films of AzAzBDI-1, AzAzBDI-2 and AzAzTTDI show better crystallinity and form larger size of microstructures by annealing, which is consistent with the enhanced device performance after thermal annealing.

Keywords azulene; nonbenzenoid aromatic hydrocarbon; imides; organic field-effect transistors; organic semiconductor

\section{1 引言}

萝(Azulene)是一种典型的非苯芳烃类化合物, 与荎 互为同分异构体. 从分子结构上看, 真可以看作是由富 电子的五元环和缺电子的七元环骈合而成(图 1a). 这种 特殊的分子结构使宷呈现青蓝色且具有较大的分子偶 极矩 $(1.08 \mathrm{D})^{[1]}$, 表现出与萗不同的物理化学性质. 萝具 有非镜面对称的前线分子轨道(HOMO/LUMO) ${ }^{[2]}$, 其苂 光主要是由第二激发态 $\left(\mathrm{S}_{2}\right)$ 到基态 $\left(\mathrm{S}_{0}\right)$ 的辐射跃迁产生 的 ${ }^{[3]}$, 不符合 Kasha 规则 $\left(\mathrm{S}_{1} \rightarrow \mathrm{S}_{0}\right)^{[4]}$; 而萗则表现出镜面 对称的前线分子轨道和符合 Kasha 规则的苂光现象. 姚 类化合物也存在于天然产物中, 表现出独特的生物活 性 ${ }^{[5]}$. 近年来, 随着有机电子学的发展, 真衍生物在有 机场效应晶体管 $(\mathrm{OFET})^{[6]}$ 、有机太阳能电池 $(\mathrm{OPV})^{[7]}$ 、 钻钣矿太阳能电池 ${ }^{[8]}$ 、非线性光学材料 ${ }^{[9]}$ 和近红外材 料 ${ }^{[10]}$ 等领域的研究受到广泛关注 ${ }^{[11]}$. 但由于合成路线

(a)

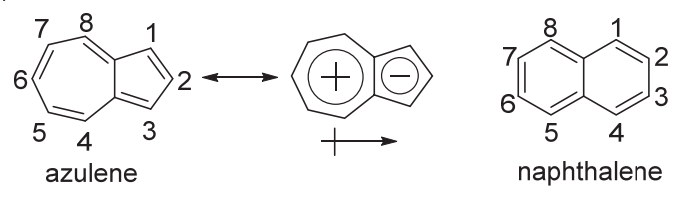

(b)<smiles>[R]N1C(=O)c2ccc3c4c(ccc(c24)C1=O)C(=O)N([R])C3=O</smiles>

$R$
1
R<smiles>CCCn1c(=O)c2ccc3c(=O)c4ccc(c1=O)c1c5ccc(c(=O)[nH]c(=O)c3c3ccc5c23)c41</smiles>

PDI

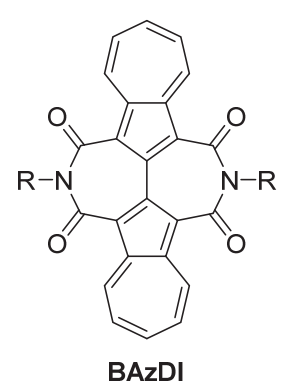

(c) This work:

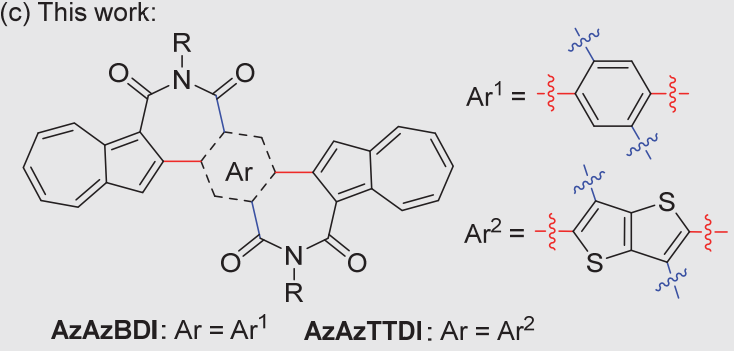

图 1 (a) 菳和菜的分子结构及原子编号; (b) 代表性酰亚胺类化合物 的分子结构; (c) AzAzBDI 和 AzAzTTDI 的分子结构

Figure 1 Molecular structures of (a) azulene and naphthalene with atom numbers, (b) representative diimides, and (c) AzAzBDI and AzAzTTDI
较长、产率较低、难以选择性官能团化等原因 ${ }^{[12]}$, 目前 基于姚的有机半导体材料的报道相对较少. 因此，奧及 其衍生物在有机材料领域具有较大的研究空间.

酰亚胺类化合物是一类重要的有机半导体材料. 自 Horowitz 等 ${ }^{[13]}$ 首次将菲二酰亚胺 (PDI) 应用于有机半导 体材料以来, 䓙二酰亚胺(NDI)、PDI 及其他酰亚胺类化 合物(图 1b)在 $\mathrm{OFET}^{[14]} 、 \mathrm{OPV}^{[15]}$ 等有机半导体领域被广 泛研究. 由于酰亚胺基团的引入能够提高材料的溶解 度、降低分子轨道能级，因此酰亚胺类化合物通常表现 出良好的可溶液加工性和优异的半导体性能 ${ }^{[16]}$. 最近, 本课题组发展了一类基于宷的酰亚胺类化合物——联 奧二酰亚胺(BAzDI) ${ }^{[17]}$, 并将其作为 $\mathrm{n}$-型有机小分子和 聚合物半导体材料应用于 OFET 和 OPV 研究 ${ }^{[18]}$. 为了 进一步研究宷酰亚胺类化合物的物理化学性质和结构性能关系, 我们发展了一种新的合成策略, 利用真各个 位点的反应活性差异, 将苯 (B) 或噻吩并 $[3,2-b]$ 噻吩 (TT)基团引入到真酰亚胺核中, 合成了苯 $(\mathbf{B})$ 或噻吩并 [3,2-b] 噻吩 (TT) 桥联的二宷二酰亚胺化合物 AzAzBDI-1、AzAzBDI-2 与 AzAzTTDI, 并研究了其相 关的物理化学性质和 OFET 性能. 与前期发展的联奧二 酰亚胺类 $\mathrm{n}$-型有机半导体不同, 这些芳基桥联的二萝二 酰亚胺化合物呈现出双极性有机半导体的特征.

\section{2 结果与讨论}

\section{1 合成与表征}

目标化合物 AzAzBDI-1、AzAzBDI-2 和 AzAzTTDI 的分子结构如图式 1 所示. 化合物 AzAzBDI-1 和 AzAzBDI-2 酰亚胺核的中间部分为苯环, $N$-烷基链分别 为 2-已基癸基和正辛基，化合物 AzAzTTDI 酰亚胺核的 中间部分为噻吩并 $[3,2-b]$ 噻吩基团, $N$-烷基链为 2-己基 癸基, 三个化合物的合成路线如图式 1 所示. 我们参照 文献的合成方法 ${ }^{[19]}$, 以商品化的环庚三烯酚酮 1 为原料 经 5 步反应得到宷硼酸酯 2 . 化合物 2 通过 Suzuki-Miyaura 反应与化合物 $\mathbf{3 a}$ 和 $\mathbf{3 b}$ 交叉偶联, 分别 以 $86 \%$ 和 $81 \%$ 的产率得到了化合物 $\mathbf{4 a}$ 和 $\mathbf{4 b}$. 由于萝的 1 -位具有强的富电子性, 化合物 $4 \mathrm{a}$ 和 $4 \mathrm{~b}$ 在三氟乙酸䣶 的作用下选择性地在 1-位发生三氟乙酰化反应, 分别以 $95 \%$ 和 $91 \%$ 的产率得到了化合物 $\mathbf{5 a}$ 和 $\mathbf{5 b}$, 随后 $\mathbf{5 a}$ 和 $\mathbf{5 b}$ 经过一步酯基、三氟乙酰基水解均以 $99 \%$ 的产率得到了 


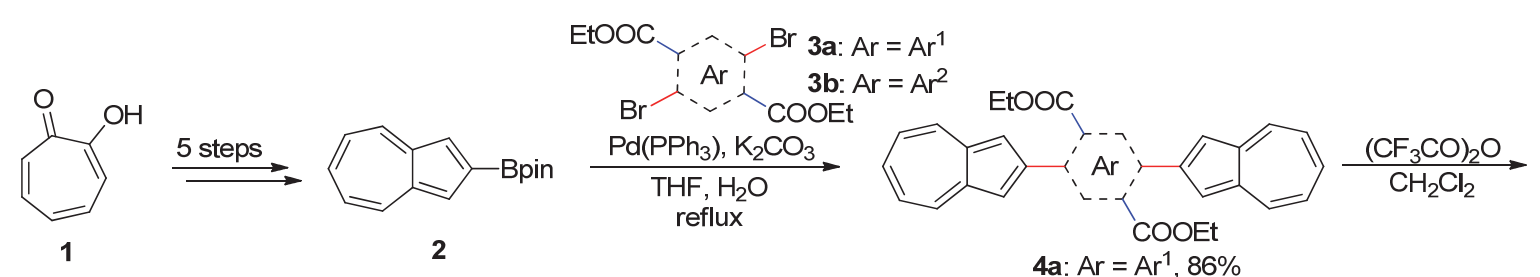

4a: $\mathrm{Ar}=\mathrm{Ar}^{1}, 86 \%$

4b: $\mathrm{Ar}=\mathrm{Ar}^{2}, 81 \%$

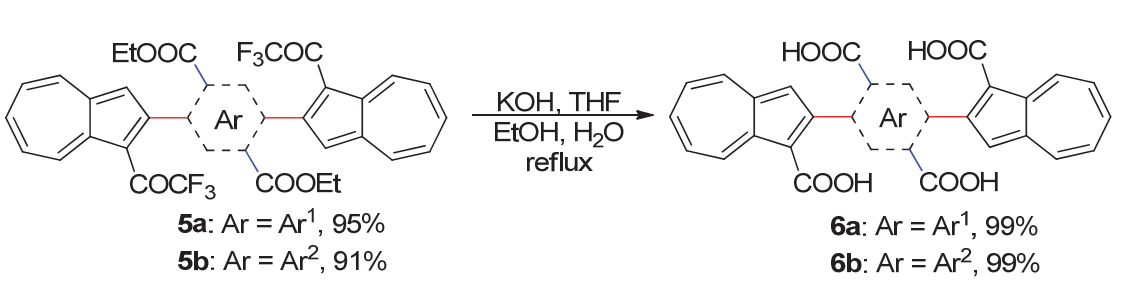

1) ${\mathrm{R}-\mathrm{NH}_{2}, \mathrm{CH}_{2} \mathrm{Cl}_{2} \text {, reflux } \mathrm{Ac} \text {, } \mathrm{NaOAc} \text {, reflux }}_{1}$

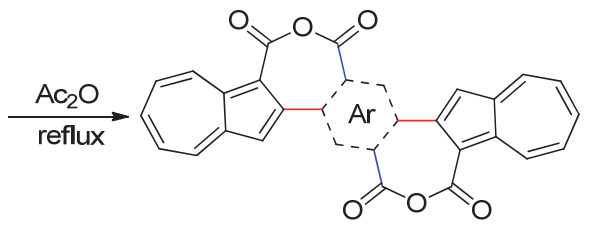

7a: $\mathrm{Ar}=\mathrm{Ar}^{1}, 88 \%$

$7 \mathrm{~b}: \mathrm{Ar}=A \mathrm{r}^{2}, 82 \%$

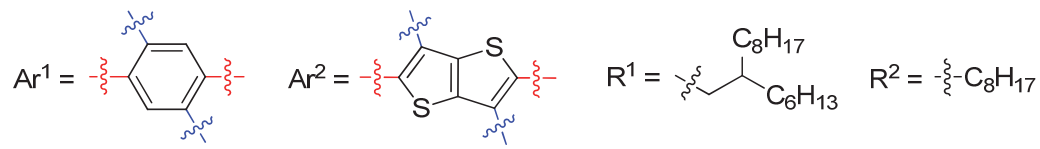

图式 1 AzAzBDI-1、AzAzBDI-2 和 AzAzTTDI 的合成路线

Scheme 1 Syntheses of AzAzBDI-1, AzAzBDI-2 and AzAzTTDI

四羧酸化合物 $6 \mathrm{a}$ 和 $6 \mathrm{~b} .6 \mathrm{a}$ 和 $6 \mathrm{~b}$ 在乙酸酐的作用下脱水 缩合分别以 $88 \%$ 和 $82 \%$ 的产率得到二酸酐化合物 $7 \mathbf{a}$ 和 7b. 最后 7a 和 $7 \mathbf{b}$ 与不同烷基链的胺经氨解、酰亚胺化 反应分别以 $58 \% 、 55 \%$ 和 $49 \%$ 的产率得到了目标化合物

AzAzBDI-1、AzAzBDI-2 和 AzAzTTDI.

我们用 ${ }^{1} \mathrm{H} N M R 、{ }^{13} \mathrm{C} N M R$ 、高分辨质谱、红外光 谱及元素分析对目标化合物 AzAzBDI-1、AzAzBDI-2 和 AzAzTTDI 的化学结构进行了表征. 在 ${ }^{1} \mathrm{H}$ NMR 谱 (图 S15、S17 和 S24)中, 化合物 AzAzBDI-1、AzAzBDI-2 和 AzAzTTDI 奧单元上 3-位氢原子的化学位移分别为 7.78、7.78 和 7.38. 由于三个化合物均具有两种化学环 境不同的羰基, 因此在红外光谱(图 S41、S42 和 S47)中, 化合物 AzAzBDI-1、AzAzBDI-2 和 AzAzTTDI 都表现 出两个不同的羰基伸缩振动峰(AzAzBDI-1: 1659, 1629 $\mathrm{cm}^{-1}$; AzAzBDI-2: 1663, $1628 \mathrm{~cm}^{-1}$; AzAzTTDI: 1637, $1598 \mathrm{~cm}^{-1}$ ). 化合物 AzAzBDI-1、AzAzBDI-2 和 AzAzTTDI 在室温条件下具有良好的溶解性, 可溶于二 氯甲烷、氯仿、四氢呋喃和甲苯等常规有机溶剂. 热重 分析(TGA)研究显示化合物 AzAzBDI-1、AzAzBDI-2 和 AzAzTTDI 失重 5\%时的热分解温度分别为 405、408 和 $417{ }^{\circ} \mathrm{C}$, 表明这三个化合物均具有良好的热稳定性. 差 示扫描量热法(DSC) 曲线显示化合物 AzAzBDI-1、

AzAzBDI-2 和 AzAzTTDI 均存在一对明显的吸放热峰, 分别对应于三个化合物的熔点和结晶点, 表明三个化合 物均具有可逆的相变过程. 由 DSC 测得的化合物
AzAzBDI-1、AzAzBDI-2 和 AzAzTTDI 的熔点分别为 173、 254 和 $275^{\circ} \mathrm{C}$, 与熔点仪的测量结果相一致.

\section{2 单晶结构和理论计算}

为了更精确地研究分子的结构和堆积方式, 我们以 四氢呋喃为溶剂, 通过溶剂缓慢挥发法得到了 AzAzBDI-2 的单晶, 并对其进行结构解析. 如图 2 所示, 单晶结构表明 AzAzBDI-2 分子具有扭曲的共轭骨架, 且在 AzAzBDI-2 分子一侧存在一个四氢呋喃分子, 形 成共晶. 两个奧环所在平面与中间苯环所在平面的二面 角分别为 $18.1^{\circ}$ 和 $23.6^{\circ}$, 两个酰亚胺基团所在平面与中 间二奧苯单元所在平面的二面角为 $23.4^{\circ}$ 和 $26.3^{\circ}$. 从堆 积图可以看出, 相邻的两个 AzAzBDI-2 分子通过分子 间宷单元缺电子的七元环和富电子的五元环之间的 $\pi-\pi$ 相互作用形成二聚体，其分子间 $\pi$-堆积距离为 0.365 $\mathrm{nm}$. 二聚体之间进一步通过面对面的 $\pi-\pi$ 相互作用(堆 积距离为 $0.355 \mathrm{~nm}$ )形成滑移的一维堆积. 这种堆积方 式与 BAzDI 系列小分子 ${ }^{[17,18 a]}$ 的堆积方式相似, 与无酰 亚胺基团的分子 ${ }^{[6]}$ 的边对面的堆积方式明显不同. 表明 酰亚胺基团的引入使分子易形成一维堆积, 有利于电荷 在分子间的传输.

为了进一步研究分子的电子性质, 我们采用 Gaussian16 软件包 ${ }^{[20]}$ 在 DFT/B3LYP/6-31G(d,p)计算水 平上对 AzAzBDI-1、AzAzBDI-2 和 AzAzTTDI 三个分 子进行结构优化, 并对其分子前线轨道的电子密度分布 
与轨道能级进行了对比分析. 如图 3 所示, AzAzBDI-1、 AzAzBDI-2 和 AzAzTTDI 三个分子均具有扭曲的共轭 骨架, 这与 AzAzBDI-2 解析的单晶结构相似. AzAzBDI-1 和 AzAzBDI-2 两个分子的 LUMO 电子密度 离域在整个分子骨架上, 而 HOMO 的电子密度主要集 中在两端的姚单元上. 对于 AzAzTTDI, 其 HOMO 和 LUMO 的电子密度均分布在整个共轭分子骨架上, 这 可能和噻吩并 $[3,2-b]$ 噻吩基团的富电子性质有关. 该结

(a)

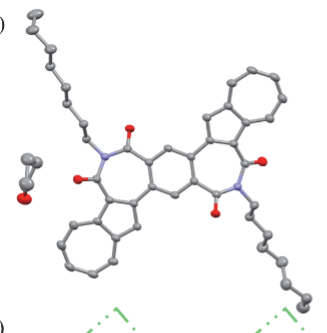

(b)
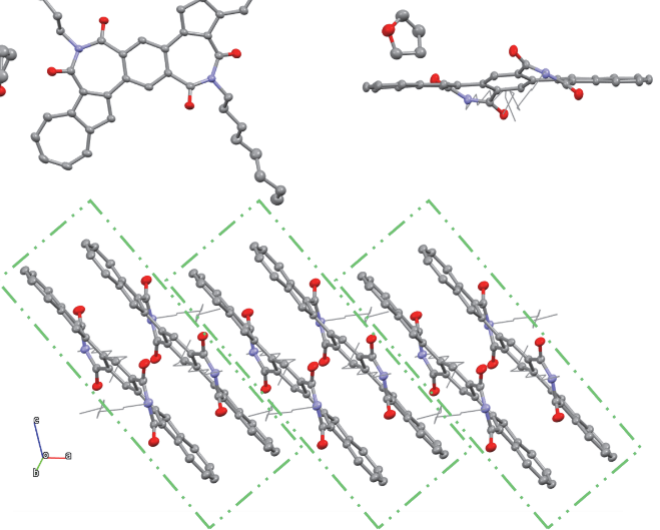

图 2 AzAzBDI-2 在单晶结构中的(a)俯视图、(b)侧视图和(c)去除 THF 分子后的分子堆积图

Figure 2 Crystal structure of AzAzBDI-2 (a) top view, (b) side view, and (c) molecular packing without THF molecules

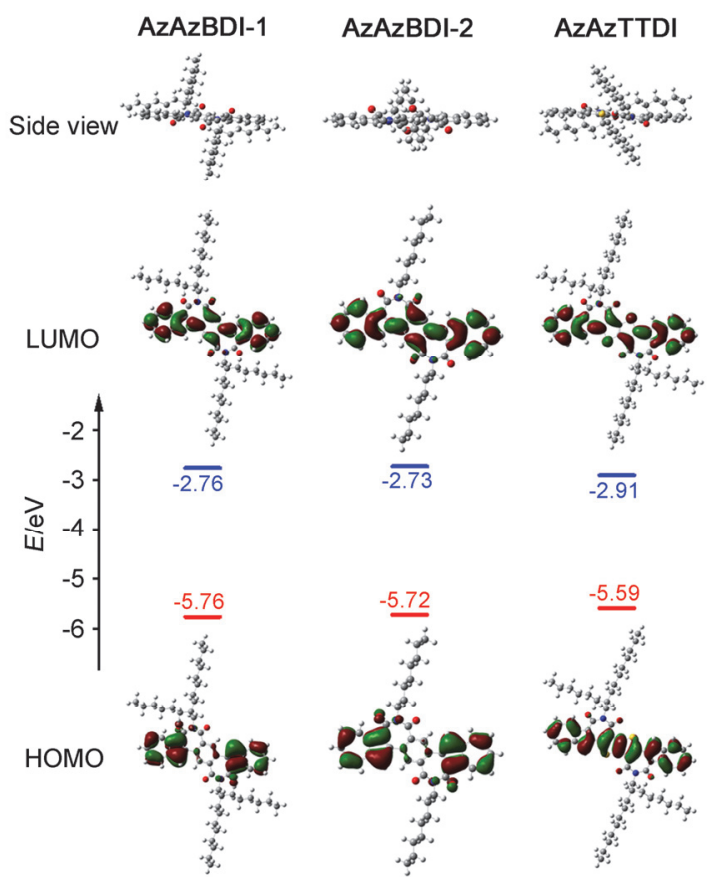

图 3 DFT 计算获得的 AzAzBDI-1、AzAzBDI-2 和 AzAzTTDI 优化 几何结构、前线轨道及轨道能级

Figure 3 Optimized molecular structure, frontier molecular orbitals and energy levels of AzAzBDI-1, AzAzBDI-2 and AzAzTTDI obtained by DFT calculations
果表明, AzAzBDI-1 和 AzAzBDI-2 有利于电子传输, 而 AzAzTTDI 既利于电子传输又利于空穴传输. 由理论计 算得到的化合物 AzAzBDI-1、AzAzBDI-2 和 AzAzTTDI 的 HOMO/LUMO 能级分别为 $-5.76 /-2.76 \mathrm{eV} 、-5.72 /$ $-2.73 \mathrm{eV}$ 和 $-5.59 /-2.91 \mathrm{eV}$, 其中 AzAzTTDI 的 HOMO-LUMO 带隙最小, 为 $2.68 \mathrm{eV}$. 根据 AzAzBDI-2 的单晶结构建模，理论模拟了室温下的晶体材料载流子 迁移率(参见 SI). 计算结果表明，沿 $a$ 轴方向可拟合出 最大的迁移率数据, 其理论最大电子和空穴迁移率分别 为 $0.025 \mathrm{~cm}^{2} \cdot \mathrm{V}^{-1} \cdot \mathrm{s}^{-1}$ 和 $0.047 \mathrm{~cm}^{2} \cdot \mathrm{V}^{-1} \cdot \mathrm{s}^{-1}$.

\section{3 光谱和电化学性质}

我们用紫外 - 可见光吸收光谱对化 合物 AzAzBDI-1、AzAzBDI-2 和 AzAzTTDI 在氯仿溶液以 及薄膜状态下的光学性质进行了研究(图 4a 和 S5), 相关 光谱数据列于表 1. AzAzBDI-1 和 AzAzBDI-2 在溶液中 的吸收光谱类似, 最强吸收峰分别为 $350 \mathrm{~nm}$ 和 $352 \mathrm{~nm}$, 在 $460 \mathrm{~nm}$ 至 $680 \mathrm{~nm}$ 之间有一宽的吸收峰，吸收强度较 弱，对应于化合物的 $\mathrm{S}_{0} \rightarrow \mathrm{S}_{1}$ 跃迁. 与 AzAzBDI-1 和
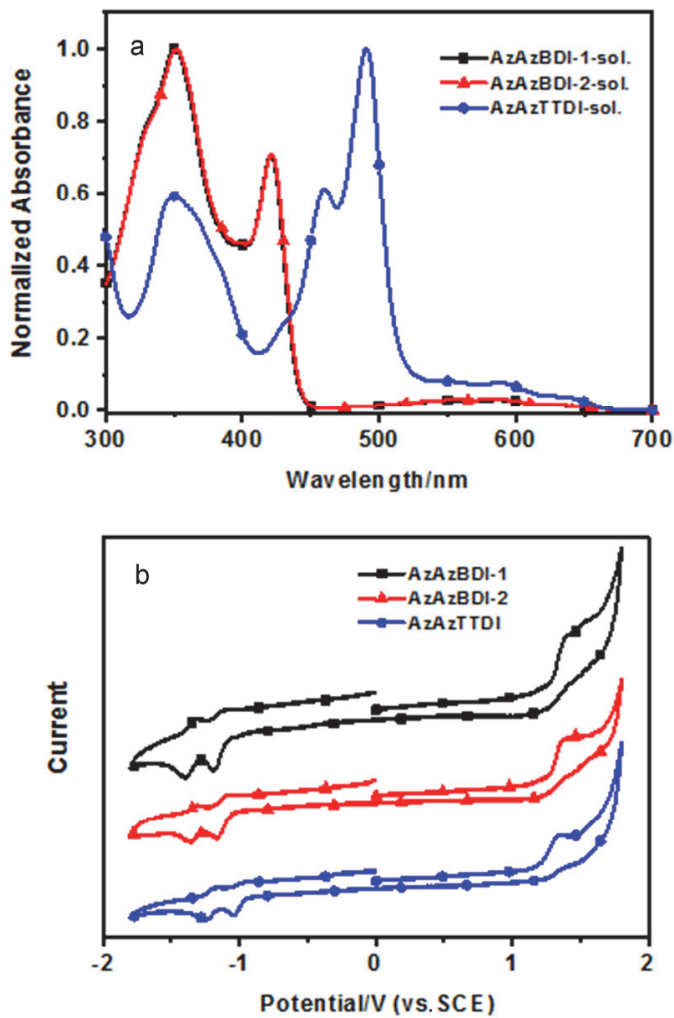

图 4 (a) AzAzBDI-1、AzAzBDI-2 和 AzAzTTDI 在氯仿溶液中的紫 外-可见吸收光谱; (b) AzAzBDI-1、AzAzBDI-2 和 AzAzTTDI 在二氯 甲烷 $\left(0.1 \mathrm{~mol} \cdot \mathrm{L}^{-1} \mathrm{Bu}_{4} \mathrm{NPF}_{6}\right.$ 作为支持电解质, 饱和甘录电极作为参比 电极, 扫描速率为 $100 \mathrm{mV} \cdot \mathrm{s}^{-1}$ )中的循环伏安曲线

Figure 4 (a) UV-vis absorption spectra of compounds AzAzBDI-1, AzAzBDI-2 and AzAzTTDI in chloroform, (b) cyclic voltammograms of AzAzBDI-1, AzAzBDI-2 and AzAzTTDI in dichloromethane $(0.1 \mathrm{~mol} \cdot$ $\mathrm{L}^{-1} \mathrm{Bu}_{4} \mathrm{NPF}_{6}$ as supporting electrolyte, $\mathrm{SCE}$ as reference electrode, scan rate of $100 \mathrm{mV} \cdot \mathrm{s}^{-1}$ ) 
表 1 AzAzBDI-1、AzAzBDI-2 和 AzAzTTDI 的光谱、电化学和理论计算数据

Table 1 Optical, electrochemical and DFT calculation data for AzAzBDI-1, AzAzBDI-2 and AzAzTTDI

\begin{tabular}{|c|c|c|c|c|c|c|c|c|c|c|c|}
\hline \multirow{2}{*}{ Compound } & \multicolumn{2}{|c|}{$\lambda_{\max } / \mathrm{nm}$} & \multirow{2}{*}{$\begin{array}{c}E_{\mathrm{g}}^{\mathrm{opt} a} / \\
\mathrm{eV}\end{array}$} & \multirow{2}{*}{$\begin{array}{c}\varphi_{\text {onset }}^{\text {red } b} / \\
\mathrm{V}\end{array}$} & \multirow{2}{*}{$\begin{array}{c}\varphi_{\text {onset }}^{\text {ox }} \quad b \\
\mathrm{~V}\end{array}$} & \multirow{2}{*}{$\begin{array}{c}E_{\mathrm{HOMO}}^{c} / \\
\mathrm{eV}\end{array}$} & \multirow{2}{*}{$\begin{array}{c}E_{\mathrm{LUMO}}{ }^{c} / \\
\mathrm{eV}\end{array}$} & \multirow{2}{*}{$\begin{array}{c}E_{\mathrm{g}}^{\mathrm{CV} d} / \\
\mathrm{eV}\end{array}$} & \multirow{2}{*}{$\begin{array}{c}E_{\mathrm{HOMO}}{ }^{e} / \\
\mathrm{eV}\end{array}$} & \multirow{2}{*}{$\begin{array}{c}E_{\mathrm{LUMO}}{ }^{e} / \\
\mathrm{eV}\end{array}$} & \multirow{2}{*}{$\begin{array}{c}E_{\mathrm{g}}^{\mathrm{Cal} d} / \\
\mathrm{eV}\end{array}$} \\
\hline & Sol. & Film & & & & & & & & & \\
\hline AzAzBDI-1 & $350,421,587$ & 349,426 & 1.82 & -1.09 & 1.28 & -5.65 & -3.28 & 2.37 & -5.76 & -2.76 & 3.00 \\
\hline AzAzBDI-2 & $352,421,585$ & $350,425,591$ & 1.82 & -1.07 & 1.28 & -5.65 & -3.30 & 2.35 & -5.72 & -2.73 & 2.99 \\
\hline AzAzTTDI & $349,460,490$ & $309,359,482$ & 1.79 & -0.95 & 1.20 & -5.57 & -3.42 & 2.15 & -5.59 & -2.91 & 2.68 \\
\hline
\end{tabular}

${ }^{a}$ Estimated from the onset absorption of the thin film; ${ }^{b}$ Onset potential versus SCE; ${ }^{c}$ Calculated from $E_{\mathrm{HOMO} / \mathrm{LUMO}}=-4.37-E_{\text {onsel }}^{\mathrm{ox}} / E_{\mathrm{onset}}^{\text {red }}($ calibration by ferrocene);

${ }^{d}$ Calculated from $\mathrm{CV} ;{ }^{e}$ Estimated from DFT calculations.

AzAzBDI-2 不同, AzAzTTDI 溶液的吸收主要集中在 410 $530 \mathrm{~nm}$, 其最大吸收峰位于 $490 \mathrm{~nm}$. 通过在石英 基底旋涂的方法得到化合物 AzAzBDI-1、AzAzBDI-2 和 AzAzTTDI 的薄膜, AzAzBDI-1、AzAzBDI-2 和 AzAzTTDI 薄膜的末端吸收分别为 683、683 和 $692 \mathrm{~nm}$, 相对于溶液的末端吸收 $(670 、 670$ 和 $667 \mathrm{~nm})$ 分别红移了 13、13 和 $25 \mathrm{~nm}$, 表明三个化合物在固态结构中呈 $J$-型 堆积. 由固态薄膜的末端吸收计算出 AzAzBDI-1、

AzAzBDI-2 和 AzAzTTDI 的光学带隙 $E_{\mathrm{g}}$ 分别为 1.82、

1.82 和 $1.79 \mathrm{eV}$.

我们采用循环伏安法 $(\mathrm{CV})$ 测试了化合物 AzAzBDI-1、AzAzBDI-2 和 AzAzTTDI 的电化学性质. 如图 $4 \mathrm{~b}$ 和表 1 所示, 三个化合物在二氯甲烷溶液中均表 现出了两对准可逆的还原峰和不可逆的氧化峰.

AzAzBDI-1、AzAzBDI-2 和 AzAzTTDI 的第一起始还 原电位分别为 $-1.09 、-1.07$ 和 $-0.95 \mathrm{~V}$, 第一起始氧化 电位分别为 $1.28 、 1.28$ 和 $1.20 \mathrm{~V}$. 在相同条件下, 测得 二茂铁 $\left(\mathrm{Fc}^{+} / \mathrm{Fc}\right)$ 相对于甘录电极的电位为 $0.43 \mathrm{~V}$. 已知 二茂铁 $\left(\mathrm{Fc}^{+} / \mathrm{Fc}\right)$ 的真空能级为 $4.80 \mathrm{eV}^{[21]}$, 根据公式 $E_{\mathrm{HOMO} / \mathrm{LUMO}}=-4.37-E_{\mathrm{oneset}}^{\mathrm{ox}} / E_{\text {oneset }}^{\mathrm{red}}$, 由第一起始氧化电位 和第一起始还原电位计算得到 AzAzBDI-1、AzAzBDI-2 和 AzAzTTDI 三个分子的 HOMO/LUMO 能级分别为 $-5.65 /-3.28 \mathrm{eV} 、-5.65 /-3.30 \mathrm{eV}$ 和 $-5.57 /-3.42 \mathrm{eV}$. 化合物 AzAzTTDI 比 AzAzBDI-1 和 AzAzBDI-2 具有更 高的 HOMO 能级和更低的 LUMO 能级, 有利于电子和 空穴的注入, 因此基于化合物 AzAzTTDI 的 OFET 器件 有望获得更高性能. 由电化学测得的三个化合物 HOMO/LUMO 能级与理论计算得到的结果相一致, 表 明三个化合物都可能具有双极性电荷传输的性质.

\subsection{OFET 器件及薄膜形貌表征}

为了研究 AzAzBDI-1、AzAzBDI-2 和 AzAzTTDI 三个化合物的电荷传输性能, 我们用溶液旋涂的方法 (参见 SI)制备了三个化合物的薄膜 OFET 器件, 研究其 电荷传输性能. AzAzBDI-1、AzAzBDI-2 和 AzAzTTDI 三个化合物 OFET 器件的输出和转移曲线分别如图 5 和 S6 所示, 相关数据总结于表 2. 器件测试结果表明三个 化合物均表现出了以 $\mathrm{n}$-型为主的双极性半导体特征, 这 与前面 DFT 计算和 CV 测试的结果相一致. 随着薄膜退 火温度的升高, 器件性能逐渐提高. AzAzBDI-1 和 AzAzBDI-2 的 OFET 器件最高电子迁移率分别为 0.068 $\mathrm{cm}^{2} \cdot \mathrm{V}^{-1} \cdot \mathrm{s}^{-1}$ 和 $0.086 \mathrm{~cm}^{2} \cdot \mathrm{V}^{-1} \cdot \mathrm{s}^{-1}$, 最高空穴迁移率分别 为 $3.1 \times 10^{-4} \mathrm{~cm}^{2} \cdot \mathrm{V}^{-1} \cdot \mathrm{s}^{-1}$ 和 $1.5 \times 10^{-3} \mathrm{~cm}^{2} \cdot \mathrm{V}^{-1} \cdot \mathrm{s}^{-1}$, 这两 个化合物 OFET 性能的差异可能是由于烷基链的结构对 分子堆积的影响造成的 ${ }^{[22]}$. 溶液法制备的化合物 AzAzBDI-2 的器件性能(电子迁移率大于空穴迁移率)与 理论计算结果(空穴迁移率大于电子迁移率)相反, 而 AzAzBDI-2 单晶器件(参见 SI) 与理论计算结果一致. 因 此, 器件的制备方法和活性层的成膜方式会影响该类化 合物的半导体性能. 与 AzAzBDI-1 和 AzAzBDI-2 的 OFET 器件相比, AzAzTTDI 的 OFET 器件表现出更优异 的半导体性能, 其最高电子和空穴迁移率分别为 0.087 $\mathrm{cm}^{2} \cdot \mathrm{V}^{-1} \cdot \mathrm{s}^{-1}$ 和 $8.8 \times 10^{-3} \mathrm{~cm}^{2} \cdot \mathrm{V}^{-1} \cdot \mathrm{s}^{-1}$, 这与前面循环伏 安法测得的 AzAzTTDI 具有更高的 HOMO 能级和更低 的 LUMO 能级的实验结果相一致, 表明噻吩并 $[3,2-b]$ 噻 吩桥联单元相对于苯环在该类酰亚胺化合物中更有利 于获得高性能的双极性有机半导体. 此外, 与无酰亚胺 基团的分子 ${ }^{[6 c]}$ 相比，酰亚胺基团的引入可以改善成膜工 艺, 并改变材料的载流子传输类型.

为了探究薄膜结构形貌与 OFET 器件性能之间的关 系, 我们用 $\mathrm{X}$ 射线衍射(XRD)和原子力显微镜(AFM)对 AzAzBDI-1、AzAzBDI-2 和 AzAzTTDI 三个化合物的 薄膜微观结构进行表征。如图 S8 所示, 化合物 AzAzBDI-1、AzAzBDI-2 和 AzAzTTDI 新制备的薄膜 XRD 图表现出一级衍射峰, 随着薄膜退火温度的升高, 三个化合物薄膜的衍射峰强度均有所增强，表明其薄膜 的结晶性得到改善, 这与 OFET 器件性能随薄膜退火温 度的升高而升高的趋势相一致. AFM 图(图 S9)显示, 当 升高退火温度时, AzAzBDI-1、AzAzBDI-2 和 AzAzTTDI 三个化合物薄膜的晶粒尺寸明显增大，对载 流子在晶粒内的传输有利, 其中化合物 AzAzTTDI 比相 同烷基链的 AzAzBDI-1 表现出更强的结晶性，这可能 是 AzAzTTDI 具有更优半导体性能的原因之一. 三个化 合物薄膜的晶界较多, 连续性较差, 阻碍了晶粒间的电 荷传输. 因此, 通过优化成膜条件, 提高薄膜的连续性 有望进一步提升化合物的 OFET 器件性能.

\section{3 结论}

我们发展了一种新的合成策略,利用奧各位点的反 应活性差异选择性地在宷的 1-位进行官能团化, 合成了 三个芳基桥连的二宷二酰亚胺化合物 AzAzBDI-1、 

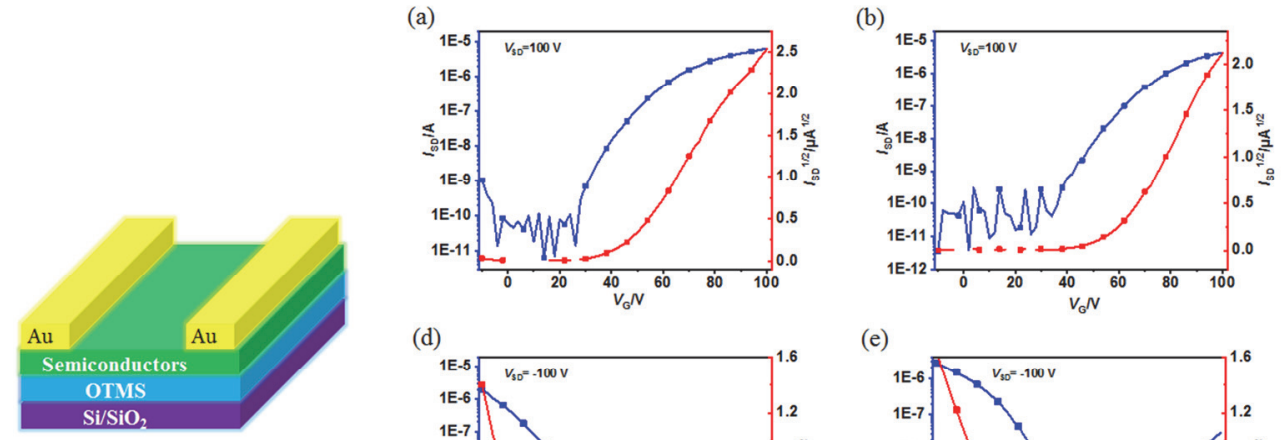

(c)
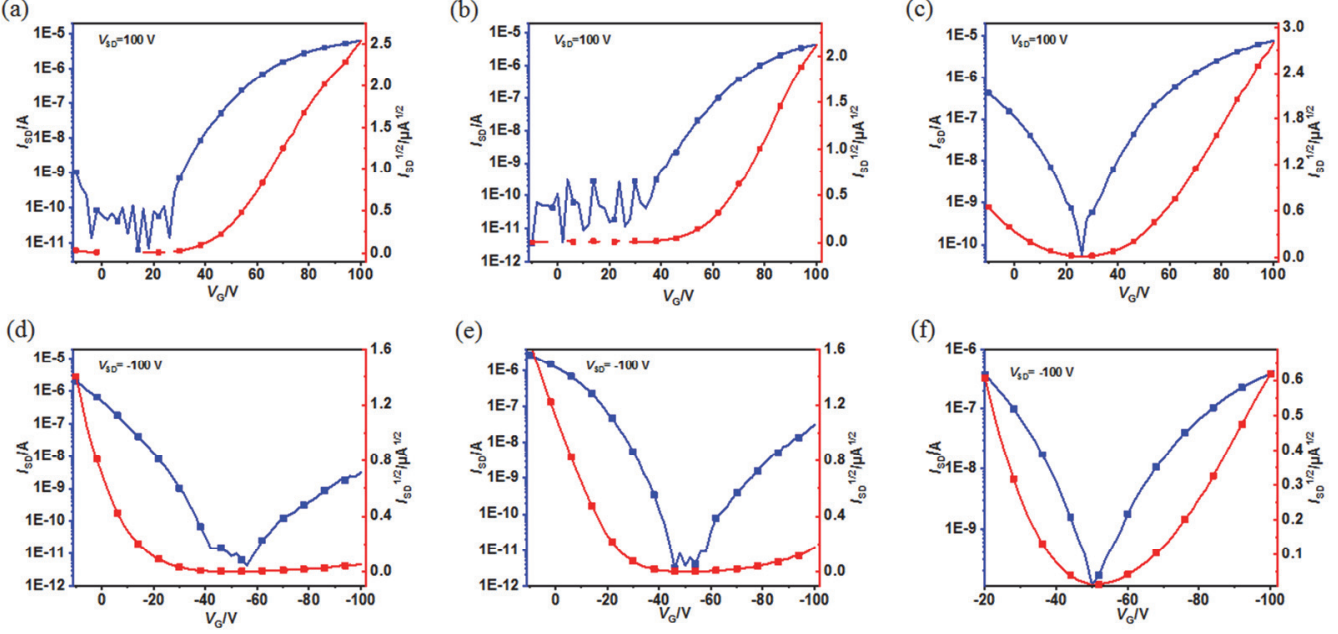

图 5 OFET 器件结构和基于(a, d) AzAzBDI-1、(b, e) AzAzBDI-2 和(c, f) AzAzTTDI 的 OFET 器件转移曲线

Figure 5 Device structure of OFET and transfer curves of OFETs based on (a, d) AzAzBDI-1, (b, e) AzAzBDI-2 and (c, f) AzAzTTDI

表 2 AzAzBDI-1、AzAzBDI-2 和 AzAzTTDI 的薄膜在不同退火温度下的 OFET 器件性能参数

Table 2 Parameters of OFET thin-film devices based on AzAzBDI-1, AzAzBDI-2 and AzAzTTDI at different thermal annealing temperatures $\left(T_{\mathrm{a}}\right)$

\begin{tabular}{|c|c|c|c|c|c|c|c|c|}
\hline Compound & $T_{\mathrm{a}} /{ }^{\circ} \mathrm{C}$ & Polarity & $\begin{array}{l}\mu_{\mathrm{e}, \text { ave }}\left(\mu_{\mathrm{e}, \max }\right)^{a} / \\
\left(\mathrm{cm}^{2} \cdot \mathrm{V}^{-1} \cdot \mathrm{s}^{-1}\right)\end{array}$ & $V_{\mathrm{T}}^{a} / \mathrm{V}$ & $I_{\mathrm{on}} / I_{\mathrm{off}}^{a}$ & $\begin{array}{l}\mu_{\mathrm{h}, \text { ave }}\left(\mu_{\mathrm{h}, \max }\right)^{a} / \\
\left(\mathrm{cm}^{2} \cdot \mathrm{V}^{-1} \cdot \mathrm{s}^{-1}\right)\end{array}$ & $V_{\mathrm{T}}^{a} / \mathrm{V}$ & $I_{\mathrm{on}} / I_{\mathrm{off}}{ }^{a}$ \\
\hline \multirow{3}{*}{ AzAzBDI-1 } & As-spun & \multirow{3}{*}{ Ambipolar } & $2.1 \times 10^{-3}\left(2.5 \times 10^{-3}\right)$ & $58 \sim 62$ & $10^{4} \sim 10^{6}$ & $3.0 \times 10^{-5}\left(4.3 \times 10^{-5}\right)$ & $-73 \sim-75$ & $10^{3} \sim 10^{5}$ \\
\hline & 80 & & $4.9 \times 10^{-3}\left(6.6 \times 10^{-3}\right)$ & $58 \sim 64$ & $10^{4} \sim 10^{6}$ & $8.8 \times 10^{-5}\left(1.4 \times 10^{-4}\right)$ & $-64 \sim-67$ & $10^{3} \sim 10^{5}$ \\
\hline & 120 & & $0.056(0.068)$ & $46 \sim 58$ & $10^{5} \sim 10^{7}$ & $1.5 \times 10^{-4}\left(3.1 \times 10^{-4}\right)$ & $-67 \sim-68$ & $10^{2} \sim 10^{3}$ \\
\hline \multirow{3}{*}{ AzAzBDI-2 } & As-spun & \multirow{3}{*}{ Ambipolar } & $0.014(0.018)$ & $69 \sim 72$ & $10^{5} \sim 10^{6}$ & $5.4 \times 10^{-5}\left(6.7 \times 10^{-5}\right)$ & $-75 \sim-79$ & $10^{3} \sim 10^{5}$ \\
\hline & 120 & & $0.064(0.086)$ & $58 \sim 68$ & $10^{4} \sim 10^{7}$ & $1.7 \times 10^{-4}\left(2.2 \times 10^{-4}\right)$ & $-72 \sim-76$ & $10^{3} \sim 10^{5}$ \\
\hline & 160 & & $0.047(0.059)$ & $42 \sim 55$ & $10^{5} \sim 10^{7}$ & $8.3 \times 10^{-4}\left(1.5 \times 10^{-3}\right)$ & $-70 \sim-73$ & $10^{3} \sim 10^{5}$ \\
\hline \multirow{4}{*}{ AzAzTTDI } & As-spun & \multirow{4}{*}{ Ambipolar } & $0.018(0.030)$ & $54 \sim 60$ & $10^{4} \sim 10^{6}$ & $8.1 \times 10^{-4}\left(1.6 \times 10^{-3}\right)$ & $-56 \sim-62$ & $10^{4} \sim 10^{6}$ \\
\hline & 120 & & $0.045(0.053)$ & $54 \sim 60$ & $10^{5} \sim 10^{7}$ & $4.8 \times 10^{-3}\left(6.3 \times 10^{-3}\right)$ & $-51 \sim-60$ & $10^{4} \sim 10^{6}$ \\
\hline & 160 & & $0.069(0.079)$ & $52 \sim 59$ & $10^{4} \sim 10^{7}$ & $7.3 \times 10^{-3}\left(8.8 \times 10^{-3}\right)$ & $-53 \sim-61$ & $10^{3} \sim 10^{6}$ \\
\hline & 200 & & $0.075(0.087)$ & $48 \sim 53$ & $10^{4} \sim 10^{6}$ & $6.1 \times 10^{-3}\left(7.7 \times 10^{-3}\right)$ & $-56 \sim-60$ & $10^{2} \sim 10^{4}$ \\
\hline
\end{tabular}

${ }^{a}$ Measured under nitrogen atmosphere and calculated from at least 15 devices.

AzAzBDI-2 和 AzAzTTDI, 其 中 AzAzBDI-1 和 AzAzBDI-2 为苯环桥联的化合物, AzAzTTDI 为噻吩并 [3,2-b]噻吩基团桥联的化合物. 单晶结构解析表明 AzAzBDI-2 具有扭曲的共轭骨架, 相邻的两个分子通 过分子间姚的七元环和五元环的 $\pi-\pi$ 相互作用形成二聚 体, 二聚体与二聚体之间通过强的 $\pi-\pi$ 相互作用形成滑 移的一维堆积. 利用 TGA、DSC、DFT 计算、紫外-可 见吸收光谱和循环伏安法等手段对三个化合物热力学 性质、光谱和电化学性质进行了研究. 通过旋涂的方法 构筑了三个化合物的 OFET 器件, 测试结果表明三个 化合物均表现出了以 $\mathrm{n}$-型为主的双极性半导体特征. 其 中 AzAzTTDI 表现出最高的 OFET 性能, 其电子和空穴 迁移率分别为 $0.087 \mathrm{~cm}^{2} \cdot \mathrm{V}^{-1} \cdot \mathrm{s}^{-1}$ 和 $8.8 \times 10^{-3} \mathrm{~cm}^{2} \cdot \mathrm{V}^{-1}$. $\mathrm{s}^{-1}$. 研究表明该类芳基桥联的二苒二酰亚胺化合物作 为双极性有机半导体具有应用潜力, 其合成策略和分子 设计思路为发展新型宷基有机半导体材料奠定了研究 基础.

\section{4 实验部分}

2-(4,4,5,5-四甲基-1,3,2-二氧杂硼烷-2-基)奧(2)根据 文献报道方法 ${ }^{[19]}$, 由环庚三烯酚酮(1)经 5 步反应制得.

2,5-二(奧-2-基)对苯二甲酸二乙酯(4a): 取 2,5-二溴 对苯二甲酸二乙酯 $\mathbf{3 a}(1.00 \mathrm{~g}, 2.63 \mathrm{mmol})$ 、化合物 $\mathbf{2}$ $(1.47 \mathrm{~g}, 5.79 \mathrm{mmol})$ 和 $\mathrm{Pd}\left(\mathrm{PPh}_{3}\right)_{4}(304 \mathrm{mg}, 26.3 \mathrm{mmol} \%)$ 于 $100 \mathrm{~mL}$ Schlenk 管中, 抽换 $\mathrm{N}_{2}$ 三次, 在氮气氛围下加入 $30 \mathrm{~mL}$ THF 和 $16 \mathrm{~mL} 1 \mathrm{~mol} \cdot \mathrm{L}^{-1} \mathrm{~K}_{2} \mathrm{CO}_{3}$ 溶液, $80{ }^{\circ} \mathrm{C}$ 加热 回流 $13 \mathrm{~h}$. 冷却至室温后, 将反应液倒入水中, $\mathrm{CH}_{2} \mathrm{Cl}_{2}$ 萃取, 无水 $\mathrm{Na}_{2} \mathrm{SO}_{4}$ 干燥, 浓缩, 柱层析 $(V(\mathrm{PE}) /$ $V\left(\mathrm{CH}_{2} \mathrm{Cl}_{2}\right)=1: 1$ ) 得 $1.07 \mathrm{~g}$ 绿色固体 $4 \mathrm{a}$, 产率 $86 \%, R_{f}$ $=0.50\left(V(\mathrm{PE}) / V\left(\mathrm{CH}_{2} \mathrm{Cl}_{2}\right)=3:\right.$ 2). m.p. $203 \sim 205{ }^{\circ} \mathrm{C} ;{ }^{1} \mathrm{H}$ NMR (400 MHz, $\left.\mathrm{CDCl}_{3}\right) \delta: 8.34(\mathrm{~d}, J=9.2 \mathrm{~Hz}, 4 \mathrm{H}), 8.05$ (s, 2H), 7.58 (d, $J=9.9 \mathrm{~Hz}, 2 \mathrm{H}), 7.50$ (s, 4H), 7.21 (t, $J=$ $9.8 \mathrm{~Hz}, 4 \mathrm{H}), 4.23(\mathrm{q}, J=7.1 \mathrm{~Hz}, 4 \mathrm{H}), 1.07$ (t, $J=7.1 \mathrm{~Hz}$, $6 \mathrm{H}) ;{ }^{13} \mathrm{C}$ NMR $\left(126 \mathrm{MHz}, \mathrm{CDCl}_{3}\right) \delta: 169.0,148.3,140.6$, 137.4, 136.9, 136.4, 134.2, 132.3, 123.8, 117.4, 61.6, 13.9; IR (KBr) v: 3075, 2979, 2904, 1720, 1571, 1537, 1474, 
$1460,1403,1379,1367,1287,1304,1264,1229,1099$, $1058,1033,947,907,843,824,775,731,677,635,582$, 532, $454 \mathrm{~cm}^{-1}$; HRMS (DART-FT) $\mathrm{m} / z:[\mathrm{M}+\mathrm{H}]^{+}$calcd for $\mathrm{C}_{32} \mathrm{H}_{27} \mathrm{O}_{4} 475.1904$, found 475.1904 .

2,5-二(宷-2-基)噻吩并 $[3,2-b]$ 噻吩-3,6-二羧酸二乙 酯 $(4 \mathbf{b})$ : 以 2,5 -二澳噻吩并 $[3,2-b]$ 噻吩-3,6-羧酸二乙酯 $(100 \mathrm{mg}, 0.23 \mathrm{mmol})$ 和化合物 $2(126 \mathrm{mg}, 0.50 \mathrm{mmol})$ 为 原料, 合成方法同化合物 $\mathbf{4 a}$, 重结晶 $\left(\mathrm{CH}_{2} \mathrm{Cl}_{2} / \mathrm{EtOH}\right)$ 得 $98 \mathrm{mg}$ 绿色固体 $\mathbf{4 b}$, 产率 $81 \%, R_{f}=0.33$ $\left(V(\mathrm{PE}) / V\left(\mathrm{CH}_{2} \mathrm{Cl}_{2}\right)=1: 1\right)$. m.p. $270 \sim 271{ }^{\circ} \mathrm{C} ;{ }^{1} \mathrm{H}$ NMR $\left(400 \mathrm{MHz}, \mathrm{CDCl}_{3}\right) \delta: 8.34(\mathrm{~d}, J=9.7 \mathrm{~Hz}, 4 \mathrm{H}), 7.83(\mathrm{~s}$, $4 \mathrm{H}), 7.58(\mathrm{t}, J=9.9 \mathrm{~Hz}, 2 \mathrm{H}), 7.19(\mathrm{t}, J=9.6 \mathrm{~Hz}, 4 \mathrm{H}), 4.46$ (q, $J=7.0 \mathrm{~Hz}, 4 \mathrm{H}), 1.45(\mathrm{t}, J=7.2 \mathrm{~Hz}, 6 \mathrm{H}) ;{ }^{13} \mathrm{C}$ NMR $(126$ $\left.\mathrm{MHz}, \mathrm{CDCl}_{3}\right) \delta: 162.4,150.4,141.2,140.4,139.9,138.0$, 137.7, 124.2, 120.3, 118.8, 61.5, 14.5; IR (KBr) v: 2979, 2906, 1722, 1680, 1572, 1533, 1500, 1483, 1464, 1438, $1405,1388,1311,1293,1267,1241,1215,1204,1168$, $1118,1043,1021,953,930,900,838,815,779,760,737$, $662,607,591,575,520,499,418 \mathrm{~cm}^{-1}$; HRMS (MALDI-TOF) $m / z$ : $[\mathrm{M}]^{+}$calcd for $\mathrm{C}_{32} \mathrm{H}_{24} \mathrm{O}_{4} \mathrm{~S}_{2}$ 536.1111, found 536.1111

2,5-二[1-(2,2,2-三氟乙酰基)萝-2-基)]对苯二甲酸二 乙酯(5a): 取化合物 $4 \mathbf{a}(1.02 \mathrm{~g}, 2.15 \mathrm{mmol})$ 于 $250 \mathrm{~mL}$ 反

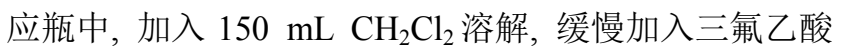
酲(5.5 mL, $38.7 \mathrm{mmol}$ ), 室温反应 $0.5 \mathrm{~h}$. 加水淬灭反应, $\mathrm{CH}_{2} \mathrm{Cl}_{2}$ 萃取, 无水 $\mathrm{Na}_{2} \mathrm{SO}_{4}$ 干燥, 浓缩, 柱层析 $\left(\mathrm{CH}_{2} \mathrm{Cl}_{2}\right)$ 得 $1.36 \mathrm{~g}$ 紫红色固体 $\mathbf{5 a}$, 产率 $95 \%, R_{f}=0.58\left(\mathrm{CH}_{2} \mathrm{Cl}_{2}\right)$. m.p. $253 \sim 254{ }^{\circ} \mathrm{C} ;{ }^{1} \mathrm{H}$ NMR $\left(400 \mathrm{MHz}, \mathrm{CDCl}_{3}\right) \delta$ : 9.46 (d, $J=10.0 \mathrm{~Hz}, 2 \mathrm{H}), 8.53(\mathrm{~d}, J=9.4 \mathrm{~Hz}, 2 \mathrm{H}), 8.08(\mathrm{~s}, 2 \mathrm{H})$, $7.94(\mathrm{t}, J=9.8 \mathrm{~Hz}, 2 \mathrm{H}), 7.77$ (t, $J=9.9 \mathrm{~Hz}, 2 \mathrm{H}), 7.66$ (t, $J$ $=9.6 \mathrm{~Hz}, 2 \mathrm{H}), 7.35(\mathrm{~s}, 2 \mathrm{H}), 4.07(\mathrm{q}, J=7.1 \mathrm{~Hz}, 4 \mathrm{H}), 0.97$ (t, $J=7.1 \mathrm{~Hz}, 6 \mathrm{H}) ;{ }^{13} \mathrm{C}$ NMR $\left(126 \mathrm{MHz}, \mathrm{CDCl}_{3}\right) \delta: 178.7$ (q, $J=36.2 \mathrm{~Hz}), 165.8,152.8,144.5,143.8,140.2,138.9$, $138.8,138.3,133.1,132.3,131.1,129.9,122.2,118.9$, $116.6(\mathrm{q}, J=290.5 \mathrm{~Hz}), 61.5,13.8 ;{ }^{19} \mathrm{~F}$ NMR $(376 \mathrm{MHz}$, $\left.\mathrm{CDCl}_{3}\right) \delta:-71.34$ (s, 6F); IR (KBr) $v: 2988,1720,1653$, 1580, 1538, 1449, 1422, 1404, 1370, 1359, 1296, 1262, 1231, 1197, 1176, 1142, 1102, 1062, 1044, 970, 920, 908, $888,866,848,810,783,758,738,725,708,681,652,617$, $576,539,520,427,412 \mathrm{~cm}^{-1}$; HRMS (MALDI-TOF) $\mathrm{m} / \mathrm{z}$ : $[\mathrm{M}+\mathrm{Na}]^{+}$calcd for $\mathrm{C}_{36} \mathrm{H}_{24} \mathrm{O}_{6} \mathrm{~F}_{6} \mathrm{Na}$ 689.1369, found 689.1380 .

2,5-二[1-(2,2,2-三氟乙酰基)宷-2-基]噻吩并 [3,2- $b$ ] 噻吩-3,6-二羧酸二乙酯(5b): 以化合物 4b $(50 \mathrm{mg}, 0.09$ $\mathrm{mmol}$ ) 为原料, 合成方法同化合物 $\mathbf{5 a}$, 重结晶 $\left(\mathrm{CH}_{2} \mathrm{Cl}_{2} / \mathrm{EtOH}\right)$ 得 $62 \mathrm{mg}$ 紫红色固体 $\mathbf{5 b}$, 产率 $91 \%, R_{f}=$ $0.67\left(\mathrm{CH}_{2} \mathrm{Cl}_{2}\right)$. m.p. $311 \sim 312{ }^{\circ} \mathrm{C}$; ${ }^{1} \mathrm{H}$ NMR $(400 \mathrm{MHz}$, $\left.\mathrm{CDCl}_{3}\right) \delta: 9.27(\mathrm{~d}, J=9.9 \mathrm{~Hz}, 2 \mathrm{H}), 8.57(\mathrm{~d}, J=9.5 \mathrm{~Hz}$,
2H), 7.95 (t, $J=9.8 \mathrm{~Hz}, 2 \mathrm{H}), 7.74$ (t, $J=9.9 \mathrm{~Hz}, 2 \mathrm{H}), 7.65$ (t, $J=9.6 \mathrm{~Hz}, 2 \mathrm{H}), 7.51(\mathrm{~s}, 2 \mathrm{H}), 4.23(\mathrm{q}, J=7.1 \mathrm{~Hz}, 4 \mathrm{H})$, $1.19(\mathrm{t}, J=7.1 \mathrm{~Hz}, 6 \mathrm{H}) ;{ }^{13} \mathrm{C}$ NMR $\left(126 \mathrm{MHz}, \mathrm{CDCl}_{3}\right) \delta$ : 179.8 (q, $J=36.0 \mathrm{~Hz}), 161.7,148.0,143.6,143.4,142.9$, $140.9,139.7,138.8,130.8,129.6,123.1,122.5,120.0$, 116.6 (q, $J=291.0 \mathrm{~Hz}), 61.6,14.0 ;{ }^{19} \mathrm{~F}$ NMR $(376 \mathrm{MHz}$, $\left.\mathrm{CDCl}_{3}\right) \delta:-71.48(\mathrm{~s}, 6 \mathrm{~F}) ; \mathrm{IR}(\mathrm{KBr}) v: 3078,1808,1702$, $1578,1527,1466,1445,1421,1380,1345,1298,1250$, 1221, 1199, 1139, 1071, 1049, 996, 899, 873, 802, 747, $707,663,623,562,506,478,419 \mathrm{~cm}^{-1}$; HRMS (MALDI-TOF) $m / z:[\mathrm{M}+\mathrm{Na}]^{+}$calcd for $\mathrm{C}_{36} \mathrm{H}_{22} \mathrm{O}_{6} \mathrm{~F}_{6} \mathrm{NaS}_{2}$ 751.0654 , found 751.0640 .

2,5-二(1-羧基姚-2-基)对苯二甲酸(6a)：取化合物 5a $(1.26 \mathrm{~g}, 1.89 \mathrm{mmol})$ 和 $\mathrm{KOH}(3.18 \mathrm{~g}, 56.7 \mathrm{mmol})$ 于 250 $\mathrm{mL}$ 反应瓶中, 加入 $30 \mathrm{~mL}$ 水、 $30 \mathrm{~mL} \mathrm{EtOH}$ 和 $30 \mathrm{~mL}$ THF 溶解, $90{ }^{\circ} \mathrm{C}$ 加热回流 $12 \mathrm{~h}$. 反应液冷却至室温后, 加入 $1 \mathrm{~mol} \cdot \mathrm{L}^{-1} \mathrm{HCl}$ 中和过量的 $\mathrm{KOH}$, 使溶液呈弱酸性, 溶液中有固体析出，过滤，干燥得 $0.95 \mathrm{~g}$ 紫红色固体 6a, 产率 99\%. m.p.>320 ${ }^{\circ} \mathrm{C}$; IR (KBr) v: 2892, 1694, 1640, 1578, 1536, 1454, 1413, 1330, 1290, 1235, 1128, 1102, 1042, 992, 958, 930, 887, 844, 821, 788, 753, 733, 674, $640,581,516 \mathrm{~cm}^{-1}$; HRMS (ESI-FTICR) $\mathrm{m} / \mathrm{z}:[\mathrm{M}-\mathrm{H}]^{-}$ calcd for $\mathrm{C}_{30} \mathrm{H}_{17} \mathrm{O}_{8}$ 505.0929, found 505.0933.

2,5 -二(1-羧基宷-2-基)噻吩并 $[3,2-b]$ 噻吩-3,6-二羧 酸 $(\mathbf{6 b})$ : 以化合物 $\mathbf{5 b}(822 \mathrm{mg}, 1.13 \mathrm{mmol})$ 为原料, 合成 方法同化合物 6a, 得 $681 \mathrm{mg}$ 绿色固体 $\mathbf{6 b}$, 产率 99\%. m.p.>320 ${ }^{\circ} \mathrm{C}$; IR (KBr) v: 2963, 1655, 1576, 1518, 1454, 1409, 1329, 1294, 1244, 1108, 1042, 988, 953, 919, 896, 886, 856, 805, 786, 734, 608, 574, 509, $456 \mathrm{~cm}^{-1}$; HRMS (MALDI-TOF) $m / z$ : $[\mathrm{M}]^{+}$calcd for $\mathrm{C}_{30} \mathrm{H}_{16} \mathrm{O}_{8} \mathrm{~S}_{2} 568.0281$, found 568.0275 .

2-(萝-2'-基)-5-(真-2"-基)苯-1,1',4,1"-四羧酸二䣶 (7a): 取化合物 6a (350 mg, $0.69 \mathrm{mmol}$ )于 $25 \mathrm{~mL}$ 反应瓶 中, 加入 $10 \mathrm{~mL}$ 乙酸酐, $150{ }^{\circ} \mathrm{C}$ 加热回流 $2 \mathrm{~h}$. 待反应液 冷却至室温后, 过滤, 干燥得 $287 \mathrm{mg}$ 棕色固体 7a, 产率 $88 \%$. m.p. $>320{ }^{\circ} \mathrm{C}$; IR (KBr) v: $3101,1737,1705,1582$, $1538,1448,1424,1393,1381,1345,1310,1296,1234$, 1217, 1200, 1137, 1088, 1072, 1046, 971, 914, 889, 820, $802,755,677,651,624,575,555,522,488,460 \mathrm{~cm}^{-1}$; HRMS (MALDI-TOF) $m / z:[\mathrm{M}+\mathrm{Na}]^{+}$calcd for $\mathrm{C}_{30} \mathrm{H}_{14} \mathrm{O}_{6} \mathrm{Na}$ 493.0683, found 493.0664.

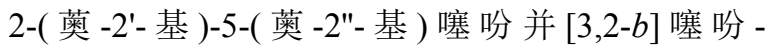
$3,1^{\prime}, 6,1^{\prime}$-四羧酸二䣶(7b): 以化合物 $\mathbf{6 b}(300 \mathrm{mg}, 0.53$ $\mathrm{mmol}$ )为原料, 合成方法同化合物 $\mathbf{7 a}$, 得 $231 \mathrm{mg}$ 棕色固 体 7b, 产率 82\%. m.p.>320 ${ }^{\circ} \mathrm{C}$; IR (KBr) v: 2988, 2956, $1725,1655,1580,1527,1442,1419,1335,1300,1267$, 1234, 1187, 1142, 1093, 1043, 1019, 966, 936, 899, 859, $841,803,794,783,760,737,725,709,647,614,595,572$, 
$524 \mathrm{~cm}^{-1}$; HRMS (MALDI-TOF) $\mathrm{m} / \mathrm{z}:[\mathrm{M}+\mathrm{H}]^{+}$calcd for $\mathrm{C}_{30} \mathrm{H}_{13} \mathrm{O}_{6} \mathrm{~S}_{2}$ 533.0148, found 533.0156.

$N, N^{\prime}$-二(2-已基癸基)[2-(英-2'-基-5-(奧-2"-基)苯1,1':4,1"-四羧酸二酰亚胺] (AzAzBDI-1): 取化合物 7a (96 mg, $0.20 \mathrm{mmol}$ )和 2-已基癸胺(148 mg, $0.61 \mathrm{mmol}$ )于 $50 \mathrm{~mL}$ 反应瓶中, 加入 $10 \mathrm{~mL} \mathrm{CH}_{2} \mathrm{Cl}_{2}$ 溶解, $50{ }^{\circ} \mathrm{C}$ 加热回 流 $12 \mathrm{~h}$. 反应液冷却至室温后, 浓缩, 得到粗产物. 再 向粗产物中加入 $\mathrm{NaOAc}(67 \mathrm{mg}, 0.81 \mathrm{mmol})$ 和 $15 \mathrm{~mL}$ 乙 酸酐, $150{ }^{\circ} \mathrm{C}$ 加热回流 $6 \mathrm{~h}$. 冷却至室温后, 将反应液浓 缩, 固体加入 $\mathrm{CH}_{2} \mathrm{Cl}_{2}$ 溶解, 水洗, 无水 $\mathrm{Na}_{2} \mathrm{SO}_{4}$ 干燥, 浓 缩, 柱层析 $\left(V(\mathrm{PE}) / V\left(\mathrm{CH}_{2} \mathrm{Cl}_{2}\right)=1: 1\right)$ 得 $109 \mathrm{mg}$ 绿色固体 AzAzBDI-1, 产率 58\%, $R_{f}=0.56\left(\mathrm{PE} / \mathrm{CH}_{2} \mathrm{Cl}_{2}, V: V=2: 1\right)$. m.p. $167 \sim 168{ }^{\circ} \mathrm{C} ;{ }^{1} \mathrm{H}$ NMR $\left(400 \mathrm{MHz}, \mathrm{CDCl}_{3}\right) \delta: 9.48$ $(\mathrm{d}, J=9.8 \mathrm{~Hz}, 2 \mathrm{H}), 8.69$ (s, 2H), $8.51(\mathrm{~d}, J=9.7 \mathrm{~Hz}, 2 \mathrm{H})$, $7.84(\mathrm{t}, J=9.8 \mathrm{~Hz}, 2 \mathrm{H}), 7.78(\mathrm{~s}, 2 \mathrm{H}), 7.59$ (t, $J=10.0 \mathrm{~Hz}$, $2 \mathrm{H}), 7.50(\mathrm{t}, J=9.6 \mathrm{~Hz}, 2 \mathrm{H}), 4.29(\mathrm{~d}, J=7.3 \mathrm{~Hz}, 4 \mathrm{H})$, $2.12 \sim 1.96(\mathrm{~m}, 2 \mathrm{H}), 1.47 \sim 1.08(\mathrm{~m}, 48 \mathrm{H}), 0.88 \sim 0.72(\mathrm{~m}$, $12 \mathrm{H}) ;{ }^{13} \mathrm{C}$ NMR $\left(126 \mathrm{MHz}, \mathrm{CDCl}_{3}\right) \delta: 169.9,166.5,143.4$, $142.9,142.6,140.0,139.1,139.0,137.3,133.9,132.2$, $128.9,127.7,119.0,115.6,51.3,37.4,32.0,31.9,31.8$, $30.3,30.0,29.7,29.5,26.51,26.49,22.8,14.3,14.2$; IR (KBr) v: 3092, 2954, 2924, 2852, 1659, 1629, 1577, 1534, 1492, 1466, 1442, 1416, 1380, 1324, 1306, 1244, 1215, 1184, 1121, 1099, 1038, 948, 922, 875, 828, 800, 766, 759, 738, 698, 648, 616, 590, 577, 544, 493, 464, $423 \mathrm{~cm}^{-1}$; HRMS (MALDI-TOF) $m / z:[\mathrm{M}+\mathrm{H}]^{+}$calcd for $\mathrm{C}_{62} \mathrm{H}_{81} \mathrm{~N}_{2} \mathrm{O}_{4}$ 917.6191, found 917.6167. Anal. calcd for $\mathrm{C}_{62} \mathrm{H}_{80} \mathrm{~N}_{2} \mathrm{O}_{4}$ : C 81.18, H 8.79, N 3.05; found C 81.02, H $8.56, \mathrm{~N} 2.88$.

$N, N^{\prime}$-二辛基[2-(宷-2'-基-5-(宷-2"-基)苯-1, 1':4,1"-四 羧酸二酰亚胺] (AzAzBDI-2): 以化合物 7a (288 mg, $0.61 \mathrm{mmol}$ )为原料, 合成方法同化合物 AzAzBDI-1, 得 $233 \mathrm{mg}$ 绿色固体 AzAzBDI-2, 产率 55\%, $R_{f}=0.47$ $\left(V(\mathrm{PE}) / V\left(\mathrm{CH}_{2} \mathrm{Cl}_{2}\right)=2: 3\right)$. m.p. $249 \sim 250{ }^{\circ} \mathrm{C} ;{ }^{1} \mathrm{H} \mathrm{NMR}$ $\left(400 \mathrm{MHz}, \mathrm{CDCl}_{3}\right) \delta: 9.55(\mathrm{~d}, J=9.9 \mathrm{~Hz}, 2 \mathrm{H}), 8.72$ (s, 2H), 8.50 (d, $J=9.8 \mathrm{~Hz}, 2 \mathrm{H}), 7.83(\mathrm{t}, J=9.8 \mathrm{~Hz}, 2 \mathrm{H}), 7.78$ (s, 2H), $7.60(\mathrm{t}, J=10.0 \mathrm{~Hz}, 2 \mathrm{H}), 7.50(\mathrm{t}, J=9.6 \mathrm{~Hz}, 2 \mathrm{H})$, $4.30(\mathrm{t}, J=6 \mathrm{~Hz}, 4 \mathrm{H}), 1.79 \sim 1.86(\mathrm{~m}, 4 \mathrm{H}), 1.49 \sim 1.17(\mathrm{~m}$, $20 \mathrm{H}), 0.86(\mathrm{t}, J=6 \mathrm{~Hz}, 6 \mathrm{H}) ;{ }^{13} \mathrm{C} \mathrm{NMR}\left(126 \mathrm{MHz}, \mathrm{CDCl}_{3}\right)$ $\delta: 169.3,165.8,143.3,142.8,142.6,139.9,139.2,138.9$, $136.7,134.1,131.9,128.9,127.7,118.4,115.8,47.5,32.0$, $29.53,29.50,28.9,27.5,22.8,14.3$; IR (KBr) v: 3440, 2921, 2851, 1663, 1628, 1577, 1534, 1490, 1465, 1444, $1417,1383,1309,1247,1211,1183,1118,1094,1039$, $947,888,865,837,809,761,732,697,665,648,623,576$, 544, 493, 436, $418 \mathrm{~cm}^{-1}$; HRMS (MALDI-TOF) $\mathrm{m} / \mathrm{z}$ : [M $+\mathrm{H}]^{+}$calcd for $\mathrm{C}_{46} \mathrm{H}_{49} \mathrm{~N}_{2} \mathrm{O}_{4}$ 693.3687, found 693.3675. Anal. calcd for $\mathrm{C}_{46} \mathrm{H}_{48} \mathrm{~N}_{2} \mathrm{O}_{4}$ : C 79.74, H 6.98, N 4.04; found $\mathrm{C} 79.80, \mathrm{H} 6.96, \mathrm{~N} 3.85$.
$N, N^{\prime}$-二(2-己基癸基)[2-(英-2'-基-5-(奧-2"-基)噻吩 并[3,2-b]噻吩-3, 1':6,1"-四羧酸二酰亚胺] (AzAzTTDI): 以化合物 7b (231 mg, $0.43 \mathrm{mmol})$ 为原料, 合成方法同化 合物 AzAzBDI-1，得 209 mg 绿色固体 AzAzTTDI，产率 $49 \%, R_{f}=0.47\left(V(\mathrm{PE}) / V\left(\mathrm{CH}_{2} \mathrm{Cl}_{2}\right)=1: 1\right)$. m.p. $271 \sim$ $272{ }^{\circ} \mathrm{C} ;{ }^{1} \mathrm{H}$ NMR (400 MHz, $\mathrm{CDCl}_{3}$ ) $\delta: 9.77$ (d, $J=9.9$ $\mathrm{Hz}, 2 \mathrm{H}), 8.22$ (d, $J=9.9 \mathrm{~Hz}, 2 \mathrm{H}), 7.56$ (t, $J=9.7 \mathrm{~Hz}, 2 \mathrm{H})$, $7.40(\mathrm{t}, J=9.9 \mathrm{~Hz}, 2 \mathrm{H}), 7.38(\mathrm{~s}, 2 \mathrm{H}), 7.31(\mathrm{t}, J=9.6 \mathrm{~Hz}$, $2 \mathrm{H}), 4.49(\mathrm{~d}, J=7.6 \mathrm{~Hz}, 4 \mathrm{H}), 2.11 \sim 2.05(\mathrm{~m}, 2 \mathrm{H}), 1.52 \sim$ $1.10(\mathrm{~m}, 48 \mathrm{H}), 0.88 \sim 0.73(\mathrm{~m}, 12 \mathrm{H}) ;{ }^{13} \mathrm{C}$ NMR $(126 \mathrm{MHz}$, $\left.\mathrm{CDCl}_{3}\right) \delta: 164.4,161.2,144.6,144.1,143.8,140.6,139.9$, $139.6,138.4,138.3,129.9,128.4,126.3,114.9,114.8$, 49.1, 36.7, 32.1, 31.9, 31.8, 30.4, 30.1, 29.8, 29.5, 26.60, 26.56, 22.9, 22.8, 14.3; IR (KBr) v: 2954, 2922, 2851, $1637,1598,1544,1527,1447,1417,1403,1377,1351$, $1327,1290,1266,1227,1199,1175,1114,1046,949,876$, 795, 755, 721, 669, 642, 629, 584, 570, 515, 443, 414 $\mathrm{cm}^{-1}$; HRMS (MALDI-TOF) $\mathrm{m} / \mathrm{z}:[\mathrm{M}+\mathrm{H}]^{+}$calcd for $\mathrm{C}_{62} \mathrm{H}_{79} \mathrm{~N}_{2} \mathrm{O}_{4} \mathrm{~S}_{2}$ 979.5476, found 979.5496. Anal. calcd for $\mathrm{C}_{62} \mathrm{H}_{78} \mathrm{~N}_{2} \mathrm{O}_{4} \mathrm{~S}_{2}$ : C 76.03, H 8.03, N 2.86; found C 75.87, $\mathrm{H}$ 8.02, N 2.69.

\section{References}

[1] (a) Wheland, G. W.; Mann, D. E. J. Chem. Phys. 1949, 17, 264. (b) Anderson, A. G.; Steckler, B. M. J. Am. Chem. Soc. 1959, 81, 4941

[2] Michl, J.; Thulstrup, E. W. Tetrahedron 1976, 32, 205.

[3] Beer, M.; Longuet-Higgins, H. C. J. Chem. Phys. 1955, 23, 1390

[4] Kasha, M. Faraday Soc. 1950, 9, 14.

[5] (a) Wang, D.; Dong, Z.; Xu, J.; Li, D. Chin. J. Org. Chem. 2013, 33 1559 (in Chinese). (王道林, 董哲, 徐姣, 李帝, 有机化学, 2013 33, 1559.) (b) Yang, F.; Wang, H.; Zhang, L.; Shi, W.; Zhang, P.; Li, Y.; Yin, S. Chin. J. Org. Chem. 2011, 31, 2106 (in Chinese). (杨芳, 王海峰, 张露昀, 石方棋, 张萍, 李颖, 尹述凡，有机化学，2011, 31, 2106.) (c) Wang, D.; Han, S.; Huang, X.; Gu, Z. Chin. J. Org. Chem. 2009, 29, 1659 (in Chinese). (王道林, 韩珊, 黄孝东, 谷峥, 有机化学, 2009, 29, 1659.) (d) Wang, D.; Xu, J.; Han, S.; Gu, Z Chin. J. Org. Chem. 2008, 28, 2016 (in Chinese). (王道林, 徐姣, 韩珊, 谷峥, 有机化学, 2008, 28, 2016.) (e)Asato, A. E.; Peng, A.; Hossain, M. Z.; Mirzadegan, T.; Bertram, J. J. Med. Chem. 1993, 36, 3137. (f) Becker, D. A.; Ley, J. J.; Echegoyen, L.; Alvarado, R. J. Am. Chem. Soc. 2002, 124, 4678.

[6] (a) Smits, E. C. P.; Setayesh, S.; Anthopoulos, T. D.; Buechel, M.; Nijssen, W.; Coehoorn, R.; Blom, P. W. M.; de Boer, B.; de Leeuw, D. M. Adv. Mater. 2007, 19, 734. (b) Wobkenberg, P. H.; Labram, J. G.; Swiecicki, J.-M.; Parkhomenko, K.; Sredojevic, D.; Gisselbrecht, J.-P.; de Leeuw, D. M.; Bradley, D. D. C.; Djukic, J.-P.; Anthopoulos, T. D. J. Mater. Chem. 2010, 20, 3673. (c) Yamaguchi, Y.; Maruya, Y.; Katagiri, H.; Nakayama, K.-i.; Ohba, Y. Org. Lett. 2012 14, 2316. (d) Yamaguchi, Y.; Ogawa, K.; Nakayama, K.-i.; Ohba, Y.; Katagiri, H. J. Am. Chem. Soc. 2013, 135, 19095. (e) Yamaguchi, Y.; Takubo, M.; Ogawa, K.; Nakayama, K.-i.; Koganezawa, T.; Katagiri, H. J. Am. Chem. Soc. 2016, 138, 11335. (f) Yao, J.; Cai, Z.; Liu, Z.; Yu, C.; Luo, H.; Yang, Y.; Yang, S.; Zhang, G.; Zhang, D. Macromolecules 2015, 48, 2039. (g) Xin, H.; Ge, C.; Fu, L.; Yang, X.; Gao, X. Chin. J. Org. Chem. 2017, 37, 711 (in Chinese). (辛涵申, 葛从伍, 傅丽娜, 杨笑迪, 高希珂, 有机化学, 2017, 37, 711.)

[7] (a) Umeyama, T.; Watanabe, Y.; Miyata, T.; Imahori, H. Chem. Lett. 2015, 44, 47. (b) Chen, Y.; Zhu, Y.; Yang, D.; Zhao, S.; Zhang, L.; Yang, L.; Wu, J.; Huang, Y.; Xu, Z.; Lu, Z. Chem. Eur. J. 2016, 22, 14527. (c) Puodziukynaite, E.; Wang, H. W.; Lawrence, J.; Wise, A J.; Russell, T. P.; Barnes, M. D.; Emrick, T. J. Am. Chem. Soc. 2014, $136,11043$.

[8] (a) Nishimura, H.; Ishida, N.; Shimazaki, A.; Wakamiya, A.; Saeki, 
A.; Scott, L. T.; Murata, Y. J. Am. Chem. Soc. 2015, 137, 15656. (b) Truong, M. A.; Lee, J.; Nakamura, T.; Seo, J. Y.; Jung, M.; Ozaki, M.; Shimazaki, A.; Shioya, N.; Hasegawa, T.; Murata, Y.; Zakeeruddin, S. M.; Grätzel, M.; Murdey, R.; Wakamiya, A. Chem. Eur. J. 2019, 25, 6741 .

[9] (a) Asato, A. E.; Liu, R. S. H.; Rao, V. P.; Cai, Y. M. Tetrahedron Lett. 1996, 37, 419. (b) Iftime, G.; Lacroix, P. G.; Nakatani, K.; Razus, A. C. Tetrahedron Lett. 1998, 39, 6853. (c) Lacroix, P. G.; Malfant, I.; Iftime, G.; Razus, A. C.; Nakatani, K.; Delaire, J. A. Chem. Eur. J. 2000, 6, 2599. (d) Coe, B. J.; Harris, J. A.; Asselberghs, I.; Clays, K.; Olbrechts, G.; Persoons, A.; Hupp, J. T.; Johnson, R. C.; Coles, S. J.; Hursthouse, M. B.; Nakatani, K. Adv. Funct. Mater. 2002, 12, 110. (e) Cristian, L.; Sasaki, I.; Lacroix, P. G.; Donnadieu, B.; Asselberghs, I.; Clays, K.; Razus, A. C. Chem. Mater. 2004, 16, 3543. (f) Migalska-Zalas, A.; El kouari, Y.; Touhtouh, S. Opt. Mater. 2012, 34, 1639. (g) Herrmann, R.; Pedersen, B.; Wagner, G.; Youn, J.-H. J. Organomet. Chem. 1998, 571, 261.

[10] (a) Kurotobi, K.; Kim, K. S.; Noh, S. B.; Kim, D.; Osuka, A. Angew. Chem., Int. Ed. 2006, 45, 3944. (b) Wang, F. K.; Lin, T. T.; He, C. B.; Chi, H.; Tang, T.; Lai, Y. H. J. Mater. Chem. 2012, 22, 10448. (c) Ince, M.; Bartelmess, J.; Kiessling, D.; Dirian, K.; Martinez-Diaz, M. V.; Torres, T.; Guldi, D. M. Chem. Sci. 2012, 3, 1472.

[11] (a) Ito, S.; Morita, N. Eur. J. Org. Chem. 2009, 4567. (b) Dong, J.; Zhang, H. Chin. Chem. Lett. 2016, 27, 1097. (c) Xin, H.; Gao, X. ChemPlusChem 2017, 82, 945. (d) Ou, L.; Zhou, Y.; Wu, B.; Zhu, L. Chin. Chem. Lett. 2019, 30, 1903.

[12] Lemal, D. M.; Goldman, G. D. J. Chem. Educ. 1988, 65, 923.

[13] Horowitz, G.; Kouki, F.; Spearman, P.; Fichou, D.; Nogues, C.; Pan, X.; Garnier, F. Adv. Mater. 1996, 8, 242.

[14] (a) Yang, N.; Qiao, X.; Fang, R.; Tao, J.; Hao, J.; Li, H. Acta Chim. Sinica 2016, 74, 335 (in Chinese). (杨宁, 乔小兰, 房忍忍, 陶竞 炜，郝健，李洪祥，化学学报, 2016, 74, 335.) (b) Yan, H.; Chen, Z.; Zheng, Y.; Newman, C.; Quinn, J. R.; Dotz, F.; Kastler, M.; Facchetti, A. Nature 2009, 457, 679. (c) Gao, X. K.; Di, C. A.; Hu, Y. B.; Yang, X. D.; Fan, H. Y.; Zhang, F.; Liu, Y. Q.; Li, H. X.; Zhu, D. B. J. Am. Chem. Soc. 2010, 132, 3697. (d) Wang, Y.; Guo, H.; Ling, S.; Arrechea-Marcos, I.; Wang, Y.; López Navarrete, J. T.; Ortiz, R. P.; Guo, X. Angew. Chem., Int. Ed. 2017, 56, 9924.

[15] (a) Jia, T.; Zheng, N.; Cai, W.; Ying, L.; Huang, F. Acta Chim. Sinica 2017, 75, 808 (in Chinese). (贾涛, 郑楠楠, 蔡万清, 应否, 黄飞, 化学学报, 2017, 75, 808.) (b) Gupta, M.; Yan, D.; Shen, F.; Xu, J.; Zhan, C. Acta Phys.-Chim. Sin. 2019, 35, 496 (in Chinese). (GUPTA Monika, 问东, 沈福刚, 徐建中, 詹传郎, 物理化学学报, 2019,
35, 496.) (c) Deng, Y.; Peng, A.; Wu, X.; Chen, H.; Huang, H. Acta Phys.-Chim. Sin. 2019, 35, 461 (in Chinese). (邓神华, 彭爱东, 吴 䈗曦, 陈华杰, 黄辉, 物理化学学报, 2019, 35, 461.) (d) Zhou, S.; Feng, G.; Xia, D.; Li, C.; Wu, Y.; Li, W. Acta Phys.-Chim. Sin. 2018 34, 344 (in Chinese). (周士超, 冯贵涛, 夏冬冬, 李诚, 武永刚, 李韦伟，物理化学学报, 2018, 34, 344.) (e) Ma, Z.; Winands, T.; Liang, N.; Meng, D.; Jiang, W.; Doltsinis, N. L.; Wang, Z. Sci. China Chem. 2020, 63, 208. (f)Liu, J.; Chen, S.; Qian, D.; Gautam, B.; Yang, G.; Zhao, J.; Bergqvist, J.; Zhang, F.; Ma, W.; Ade, H.; Inganäs, O.; Gundogdu, K.; Gao, F.; Yan, H. Nat. Energy 2016, 1, 16089.

[16] Guo, X.; Facchetti, A.; Marks, T. J. Chem. Rev. 2014, 114, 8943.

[17] Xin, H.; Ge, C.; Yang, X.; Gao, H.; Yang, X.; Gao, X. Chem. Sci. 2016, 7, 6701.

[18] (a) Xin, H.; Li, J.; Ge, C.; Yang, X.; Xue, T.; Gao, X. Mater. Chem. Front. 2018, 2, 975. (b) Xin, H.; Ge, C.; Jiao, X.; Yang, X.; Rundel, K.; McNeill, C. R.; Gao, X. Angew. Chem., Int. Ed. 2018, 57, 1322.

[19] Gao, H.; Yang, X.; Xin, H.; Gao, T.; Gong, H.; Gao, X. Chin. J. Org. Chem. 2018, 38, 2680 (in Chinese). (高洪否, 杨笑迪，辛涵申，高 铁阵, 龚和贵, 高希珂, 有机化学, 2018, 38, 2680.)

[20] Frisch, M. J.; Trucks, G. W.; Schlegel, H. B.; Scuseria, G. E.; Robb, M. A.; Cheeseman, J. R.; Scalmani, G.; Barone, V.; Petersson, G. A.; Nakatsuji, H.; Li, X.; Caricato, M.; Marenich, A. V.; Bloino, J.; Janesko, B. G.; Gomperts, R.; Mennucci, B.; Hratchian, H. P.; Ortiz, J. V.; Izmaylov, A. F.; Sonnenberg, J. L.; Williams-Young, D.; Ding, F.; Lipparini, F.; Egidi, F.; Goings, J.; Peng, B.; Petrone, A.; Henderson, T.; Ranasinghe, D.; Zakrzewski, V. G.; Gao, J.; Rega, N.; Zheng, G.; Liang, W.; Hada, M.; Ehara, M.; Toyota, K.; Fukuda, R.; Hasegawa, J.; Ishida, M.; Nakajima, T.; Honda, Y.; Kitao, O.; Nakai, H.; Vreven, T.; Throssell, K.; Montgomery, J. A., Jr.; Peralta, J. E.; Ogliaro, F.; Bearpark, M. J.; Heyd, J. J.; Brothers, E. N.; Kudin, K. N.; Staroverov, V. N.; Keith, T. A.; Kobayashi, R.; Normand, J.; Raghavachari, K.; Rendell, A. P.; Burant, J. C.; Iyengar, S. S.; Tomasi, J.; Cossi, M.; Millam, J. M.; Klene, M.; Adamo, C.; Cammi, R.; Ochterski, J. W.; Martin, R. L.; Morokuma, K.; Farkas, O.; Foresman, J. B.; Fox, D. J. Gaussian 16, Gaussian, Inc., Wallingford CT, 2016.

[21] Brown, A. R.; Jarrett, C. P.; deLeeuw, D. M.; Matters, M. Synth. Met. 1997, 88, 37.

[22] (a) Lei, T.; Dou, J.; Pei, J. Adv. Mater. 2012, 24, 6457. (b) Zhang, F.; Hu, Y.; Schuettfort, T.; Di, C. A.; Gao, X.; McNeill, C. R.; Thomsen, L. S.; Mannsfeld, C.; Yuan, W.; Sirringhaus, H.; Zhu, D. J. Am. Chem. Soc. 2013, 135, 2338. 